\title{
Programming Strain Rate Dependency into Mechanical Metamaterials
}

\author{
Sankalp Patil ${ }^{1,2 *}$ and Georg Ganzenmüller ${ }^{1,2}$ \\ ${ }^{1}$ Fraunhofer Ernst-Mach-Institute for High-Speed Dynamics, EMI, Freiburg i. Br., Germany \\ ${ }^{2}$ Institute for Sustainable Systems Engineering, INATECH, Albert-Ludwigs-Universität-Freiburg, \\ Freiburg i. Br., Germany
}

\begin{abstract}
This work presents an approach to introduce significant strain rate sensitivity into metallic metamaterials that are manufactured via additive manufacturing, where the base material employed will typically have a weak strain rate sensitivity. Here, we employ friction between the rough surfaces as the strain-rate dependent mechanism, whose magnitude is tunable by optimizing the geometry. The design along with the preliminary simulation results of the friction unit cell is presented. This work will quantify the effects of geometrical parameters on the dissipated energy.
\end{abstract}

\section{Introduction and Methodology}

Additive manufacturing of metals allows to design metamaterials specifically tailored for crash and impact applications. However, the inherent strain rate sensitivity of the base material developed for metal additive manufacturing technologies is typically weak. For maximum crashworthiness, it is desirable to not only control the structure but also the viscoelastic behaviour of the metamaterial.

This work attempts to introduce strain rate sensitivity into metallic metamaterials using friction as the governing mechanism. The magnitude of friction is tunable via the geometry. As friction between rough metallic surfaces is strain-rate dependent [1], this approach seems a promising route towards controlling strain rate sensitivity in metamaterials. This situation is in stark contrast with polymers, as these possess an inherent strong strain rate sensitivity, i.e., a non-tunable viscoelasticity which originates from their molecular structure. The idea of exploiting friction as an internal degree of freedom was initially implemented by A. Garland et.al. [2].

This work will present some preliminary simulation results and quantify the effects of several geometrical parameters pertaining to our bowtie friction unit cell design, Fig. 1. (a). The main idea is to redirect compressive loads into an orthogonal direction and utilise large frictional contact areas between the central stems and the curved beams as indicated in Fig. 1. (b), thus enhancing energy dissipation.

\footnotetext{
*Corresponding author: sankalp.patil@inatech.uni-freiburg.de
} 




Fig. 1. A bowtie unit cell equipped with friction element in its original state. The key geometrical parameters shown are to be optimized for maximum energy dissipation via friction under compressive loading (a). A simulation output image with colour fringing of von-Mises stress [GPa] under compressive load (b). The optimal set of geometrical parameters aid to efficiently exploit the frictional properties that paves a way for strain rate dependency in the structure.

\section{References}

1. L. J. Oestringer and C. Proppe, On the calculation of a dry friction coefficient, Proceedings in Applied Mathematics and Mechanics, Weinheim, Germany (2019)

2. A. Garland, K. Adstedt, Z. Casias, B. White, W. Mook, B. Kaehr, B. Jared, B. Lester, N. Leathe, E. Schwaller, B. Boyce, Coulombic friction in metamaterials to dissipate mechanical energy, Extreme Mechanics Letters (2020) 